discharge were severe disability and frailty, high risk of falls and short life expectancy.

The authors conclude that, in their study, underutilization of warfarin mostly occurred before stroke onset; by the time stroke had occurred, warfarin administration was precluded in approximately $50 \%$ of patients. They state that decisions regarding warfarin therapy in stroke patients with AF should be made on the basis of individual assessment.

Original article Somerfield J et al. (2006) Not all patients with atrial fibrillation-associated ischemic stroke can be started on anticoagulant therapy. Stroke 37: 1217-1220

\section{Fluorescence-guided surgery improves outcome in patients with malignant glioma}

A recent trial by Stummer et al. on behalf of the ALA-Glioma Study Group has shown that fluorescence-guided surgery with 5-aminolevulinic acid results in a twofold increase in the number of patients free from residual malignant glioma, detected by postoperative MRI, when compared with conventional white-light surgery.

Complete resection of glioma is challenging, as tumor margins are difficult to define. The prodrug 5-aminolevulinic acid improves visibility by producing intratumoral accumulation of fluorescent porphyrins, which can be visualized with a modified neurosurgical microscope. In this study, 322 patients with malignant glioma were randomized to undergo tumor resection with either white light or fluorescence-guided neurosurgery at one of 17 German treatment centers. MRI was performed on all patients $72 \mathrm{~h}$ postoperatively and at regular intervals during follow-up. After a median follow-up of 35.4 months, significantly more patients in the fluorescence-guided surgery group than in the white-light surgery group had complete tumor resection $(P<0.0001)$; of those with residual disease, patients in the former group had significantly smaller tumor volumes $(P<0.0001)$. Progression-free survival at 6 months was also significantly greater in patients whose tumors were resected under fluorescent light $(P=0.0003)$ than in those who underwent white-light surgery. There were no significant long-term differences in neurotoxicity or Karnofsky performance status between the two groups. The authors conclude that patients with malignant glioma derive substantial clinical benefit from fluorescence-guided tumor resection with 5-aminolevulinic acid.

Original article Stummer W et al. (2006) Fluorescenceguided surgery with 5-aminolevulinic acid for resection of malignant glioma: a randomised controlled multicentre phase III trial. Lancet Oncol 7: 392-401

\section{Resolution of migraine headaches after transcatheter closure of patent foramen ovale}

Several studies have demonstrated a strong association between patent foramen ovale (PFO) and migraine headache with aura (MHA). Preliminary data have shown substantial improvements in MHA severity after transcatheter PFO closure. Giardini and colleagues, from Bologna University in Italy, sought to establish which factors lead to MHA in patients with PFO and assess the mechanisms through which MHA is resolved after PFO closure.

Their study comprised 131 consecutive patients with PFO and at least one documented stroke. A total of $35(27 \%)$ of patients from this group were diagnosed as having MHA; the incidence and severity of their symptoms was evaluated by use of a Migraine Disability Assessment (MIDAS) questionnaire.

Patients with MHA were more likely to be female $(P=0.001)$, have a more complex atrial septal anatomy $(P=0.001)$ and have associated thrombophilia $(P=0.007)$, than those without MHA. Transesophageal echocardiography and transcranial Doppler scanning revealed that spontaneous right-to-left shunts, and large shunts, were more prevalent in patients with MHA than in those without.

For most patients with MHA (91\%), transcatheter closure of the PFO completely or substantially resolved their MHA symptoms. Furthermore, these improvements generally lasted for more than 1 year after the procedure.

These findings lend support to the theory that MHA could be triggered in patients with PFO by persistent right-to-left shunting, which permits small venous thrombus or platelet aggregate to enter the cerebral circulation. Transcatheter closure of the defect seems to resolve MHA by abolishing the right-to-left shunt.

Original article Giardini A et al. (2006) Transcatheter patent foramen ovale closure mitigates aura migraine headaches abolishing spontaneous right-to-left shunting. Am Heart J 151:922.e1-922.e5 